\title{
The effect of Valsalva maneuver in attenuating skin puncture pain during spinal anesthesia: a randomized controlled trial
}

\author{
Sanjay Kumar, Sujeet Kumar Singh Gautam, Devendra Gupta, Anil Agarwal, \\ Sanjay Dhirraj, and Sandeep Khuba \\ Department of Anesthesiology, Sanjay Gandhi Postgraduate Institute of Medical Sciences, Lucknow, India
}

\begin{abstract}
Background: Valsalva maneuver reduces pain by activating sinoaortic baroreceptor reflex arc. We planned this study to evaluate the role of valsalva in attenuating spinal needle-puncture pain.

Methods: Ninety American Society of Anesthesiologists (ASA) grade I and II enrolled patients undergoing elective surgery were randomized into 3 groups of 30 each. Group I (Control): didn't blow; group II (Distraction): patients blew into rubber tube; Group III (Valsalva): blew into sphygmomanometer tube and raise mercury column up to $30 \mathrm{mmHg}$ for at least 20 seconds. During above procedures, spinal puncture was performed with 25-gauge spinal needle.

Results: Eighty-two patient data were analyzed. Incidence of spinal puncture pain was reduced to $10 \%$ (3 of 27) in Valsalva group as compared to $100 \%$ (28 of 28 in control group and 27 of 27 in Distraction group) observed in other two groups $(\mathrm{P}<0.05)$. Severity of lumbar puncture pain as assessed by visual analog scale $(0-10$; where 0 is no pain and 10 is the worst imaginable pain) presented as Median (Interquartile range) were significantly reduced in the Valsalva group (0.0 [0.0] as compared to other 2 groups $2.0[0.0]$ in the Distraction group and $3.0[0.8]$ in Control group) $(\mathrm{P}<0.05)$. Regarding time taken by CSF to fill spinal needle hub, there was no difference among the three groups $(\mathrm{P}>0.05)$. None patient of all groups had post dural puncture headache $(\mathrm{P}>0.05)$.

Conclusions: Valsalva can be performed routinely in ASA I and II patients undergoing spinal anesthesia as it is safe, painless and non-pharmacological method of pain attenuation.
\end{abstract}

Key Words: Distraction, Lumbar puncture pain, Spinal anesthesia, Valsalva maneuver, Visual analog scale.

Corresponding author: Sanjay Kumar, M.D.

Department of Anesthesiology, Sanjay Gandhi Postgraduate Institute of Medical Sciences, Raebareli Road, Lucknow, Uttar Pradesh 226014 India

Tel: 91-9452535221, Fax: 91-522-2668017

E-mail: drsanjaygupta9@gmail.com

ORCID: http://orcid.org/0000-0003-2875-5838

Received: May 27, 2015.

Revised: 1st, June 17, 2015; 2nd, June 20, 2015; 3rd, June 23, 2015.

Accepted: June 23, 2015.

Korean J Anesthesiol 2016 February 69(1): 27-31

http://dx.doi.org/10.4097/kjae.2016.69.1.27

\section{Introduction}

Spinal needle puncture pain can be one of the distressing factors for patient undergoing spinal anesthesia leading to refusal because of fear of puncture pain and postoperative backache [1]. Spinal needle puncture pain has both somatic and psychological components.

Different methods have been described in literature to minimize spinal puncture pain. Pharmacological methods like eutectic mixture of local anesthetic (EMLA) patch, local anesthetic infiltration reduces only somatic component of pain. Local anesthetic infiltration is itself a painful procedure [2-4]. EMLA patch

(c) This is an open-access article distributed under the terms of the Creative Commons Attribution Non-Commercial License (http://creativecommons.org/ licenses/by-nc/4.0/), which permits unrestricted non-commercial use, distribution, and reproduction in any medium, provided the original work is properly cited. 
and local anesthetic infiltration both do not alleviate psychological component of pain while non-pharmacological methods like attention-diverting measures (pressing ball, audio-visual distraction) minimizes only the psychological component of pain [4-7].

Valsalva maneuver decreases both somatic and psychological component of pain associated with venous cannulation $[8,9]$. Present study was planned to evaluate the efficacy of Valsalva maneuver on the attenuation of spinal needle puncture pain.

\section{Materials and Methods}

This prospective, randomized, placebo-controlled study was conducted between July to October 2014, following institutional ethics committee approval (Ref: 2014/114/IP/78) and written informed consent from patients. The study is registered at Clinical Trial Registry of India (Ref: CTRI: 2014:09:005037).

\section{Inclusion criteria}

Ninety consecutive adult patients of American Society of Anesthesiologists (ASA) grade I and II of either sex, who underwent surgery under subarachnoid block by midline approach in sitting position, were included in the study. Patients were instructed to take absolute bed rest for 6 hours during immediate postoperative period.

\section{Exclusion criteria}

Patients with communication problems, skin problem such as infection at the site of proposed lumbar puncture, scars, eczema, history of previous neuraxial block and spine surgery, patient's refusal for subarachnoid block, patients requiring $>1$ attempt for successful spinal or change of inter vertebral space and patients who were unable to hold the mercury column up to $30 \mathrm{mmHg}$ for 20 seconds, were excluded from the study.

\section{Sample size estimation}

Calculation of sample size was done before starting this study with the presumption that therapy would reduce spinal puncture pain by $30 \%$; power analysis with $\alpha=0.05$ and $\beta=0.80$ revealed that we would need to enroll 24 patients in each group. We enrolled 30 patients in each group to take care of dropouts, if any.

\section{Randomization, study intervention and blinding}

Enrolled patients were premedicated with oral lorazepam 2 mg the night before surgery and again at 7 am on the morning of surgery with sips of water. Patients enrolled in the study were posted as first case in the operation theatre and study was done during 9-11 am each day to avoid influence of circadian difference on pain sensitivity.

Computer generated method of randomization was adopted to divide patients into three equal groups of 30 each. Group I (Control): acted as control and did not perform valsalva nor blew through an open sphygmomanometer tube as in group II and group III; Group II (Distraction): patients were asked to blow through a rubber tube not connected to sphygmomanometer for at least $20 \mathrm{sec}$; Group III (Valsalva): patients were asked to blow into a rubber tubing connected to sphygmomanometer and raise the mercury column up to $30 \mathrm{mmHg}$ for a period of at least $20 \mathrm{sec}$ (Fig. 1). Standard monitoring like electrocardiograph, $\mathrm{SpO}_{2}$, blood pressure, respiratory rate were done. After 20 sec of intervention, spinal anesthesia was performed by a $25 \mathrm{G}$ Quincke spinal needle (BD Medical, Becton Dickinson S.A., S. Agustin del Guadalix, Madrid, Spain) by a registrar (S.A.) who was neither aware of the group distribution nor part of study.

Primary objective of the study was to evaluate the efficacy of Valsalva procedure in attenuating the pain by skin puncture during spinal anesthesia, whereas the secondary objectives were to evaluate the efficacy of Valsalva procedure on CSF flow

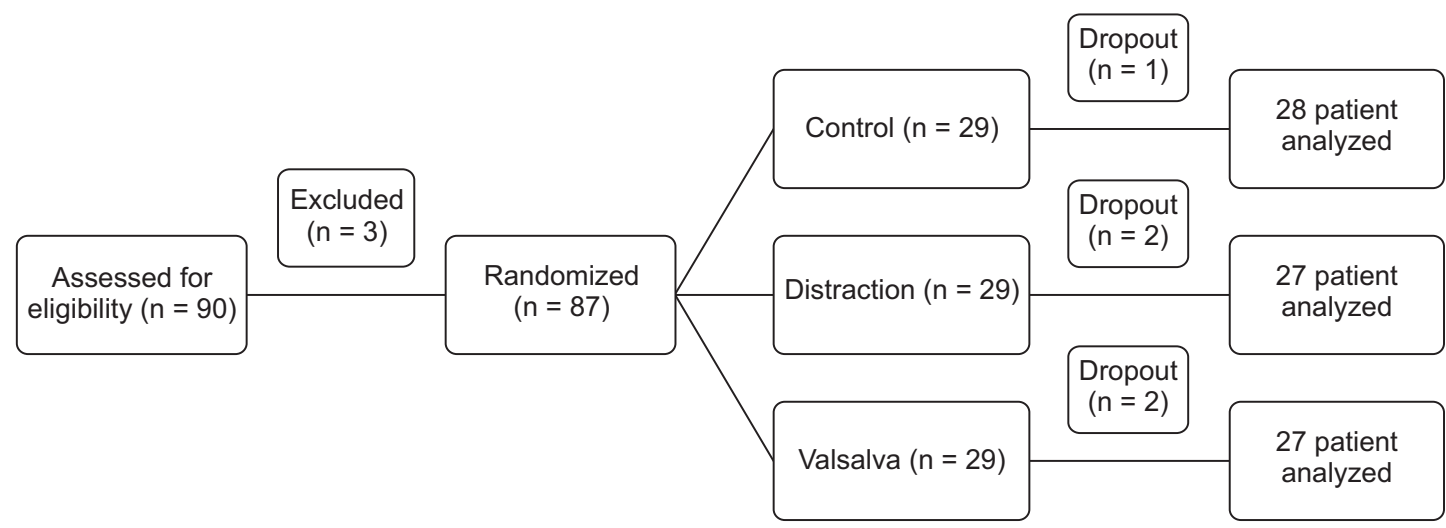

Fig. 1. Study design. 
through the spinal needle and its effect if any on the incidence of post dural puncture headache. The lumbar puncture pain was assessed by visual analog scale (VAS) score [0-10]; where 0 is no pain and 10 is the worst imaginable pain. Secondary objectives were assessed in terms of time taken by CSF to fill spinal needle hub and presence of post dural puncture headache which was assessed at 0,6 and $24 \mathrm{~h}$ following spinal puncture. Stop watch was used to record time taken by CSF to fill spinal needle hub starting from dural puncture. Both the outcomes were assessed by another anesthesia registrar (R.S.) who was blind to group allocation and was not involved in the study.

\section{Statistical analysis}

Demographic data were analyzed with one way ANOVA for continuous variables and chi square test for categorical variables. The VAS pain scores were analyzed with Mann Whitney U test; time taken by CSF to fill spinal needle hub was analyzed with ttest; incidence of PDPH was analyzed with Fisher exact test with Bonferroni correction. The method of analysis was decided prospectively and incorporated the intention-to-treat principle. The package SPSS 20.0 (IBM Corporation, New York, NY, USA). $\mathrm{P}<0.05$ was considered significant.

Table 1. Demographic Data

\begin{tabular}{lccc}
\hline & I (Control) & II (Distraction) & III (Valsalva) \\
\hline $\mathrm{N}$ & 28 & 27 & 27 \\
Age $(\mathrm{yr})$ & $42.2 \pm 13.0$ & $46.0 \pm 10.9$ & $39.1 \pm 12.9$ \\
Sex $(\mathrm{M} / \mathrm{F})$ & $14 / 14$ & $14 / 13$ & $14 / 13$ \\
Height $(\mathrm{cm})$ & $154.0 \pm 8.3$ & $153.8 \pm 8.4$ & $153.7 \pm 8.8$ \\
Weight $(\mathrm{kg})$ & $57.1 \pm 11.0$ & $59.1 \pm 9.6$ & $61.1 \pm 8.7$
\end{tabular}

Data presented either as absolute numbers or Mean \pm SD. There was no significant difference among the three groups $(\mathrm{P}>0.05)$ regarding age, sex, height and weight.

Table 2. Assessment of Spinal Puncture Pain

\begin{tabular}{cccc}
\hline & $\begin{array}{c}\text { I (Control) } \\
(\mathrm{N}=28)\end{array}$ & $\begin{array}{c}\text { II (Distraction) } \\
(\mathrm{N}=27)\end{array}$ & $\begin{array}{c}\text { III (Valsalva) } \\
(\mathrm{N}=27)\end{array}$ \\
\hline Incidence of Pain & $28 / 28$ & $27 / 27$ & $3 / 27^{*}$ \\
\hline
\end{tabular}

Data presented as absolute numbers. ${ }^{*} \mathrm{P}<0.05$ when Valsalva group was compared with other two groups.

\section{Results}

Total 90 patients were enrolled for eligibility, out of which 87 patients were posted for study following randomization and 82 patients i.e. $94 \%$ completed the study (Fig. 1). The reasons for patients not being randomized were refusal to participate in the study ( 3 patients opted for general anesthesia). Five patients were excluded from the study following initial randomization and were therefore not subjected for further analysis (2 could not perform Valsalva maneuver and 3 required second skin prick for successful dura puncture during spinal anesthesia). Total 82 patients were assessed for statistical analysis. There was no difference amongst the groups as regards to age, sex, weight and height distribution $(\mathrm{P}>0.05)$ (Table 1$)$.

Incidence of spinal puncture pain was reduced to $10 \%$ (3 of 27) in Valsalva group as compared to $100 \%$ (28 of 28 in Control group and 27 of 27 in Distraction group) observed in other two groups $(\mathrm{P}<0.05$, Table 2$)$. Severity of lumbar puncture pain as assessed by VAS, presented as Median (interquartile range) were significantly reduced in the Valsalva group $(0.0[0.0])$ as compared to other 2 groups $(2.0[0.0])$ in Distraction group and 3.0 [0.8] in Control group ( $\mathrm{P}<0.001$, Fig. 2).

Regarding time taken by CSF to fill spinal needle hub, there was no difference among the three groups ( $\mathrm{P}>0.05$, Table 3 ). None patient of all groups had post dural puncture headache $(\mathrm{P}>0.05)$.

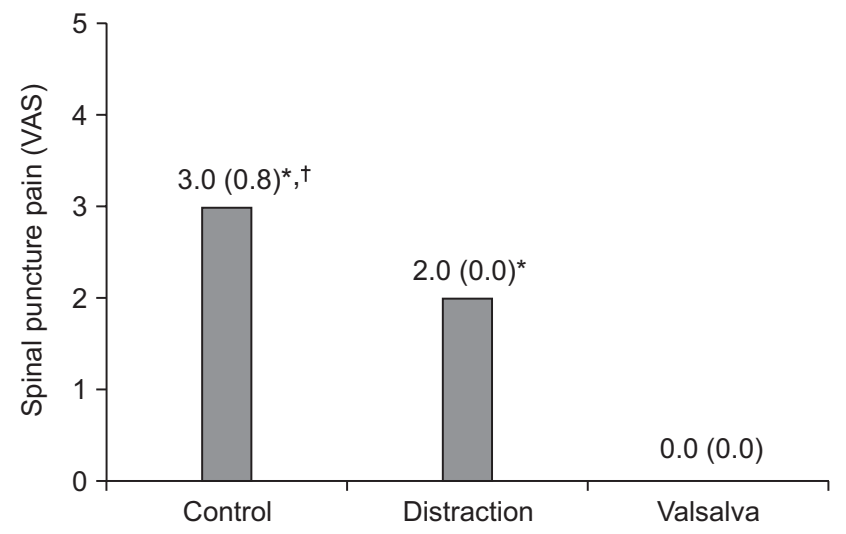

Fig. 2. Severity of spinal puncture pain. Effect of various groups on visual analog score (VAS) (median with interquartile range). $* \mathrm{P}<0.05$ when Valsalva group was compared with other two groups. ${ }^{\dagger} \mathrm{P}<0.05$ when Distraction group was compared with Control group.

Table 3. Time Taken by CSF to Fill Needle Hub

\begin{tabular}{lcc}
\hline & I (Control) & II (Distraction) \\
\hline Time to fill needle hub (sec) & $12.2 \pm 2.3$ & $13.1 \pm 2.4$ \\
\hline
\end{tabular}

Value presented as Mean \pm SD. There was no significant difference among three groups in time taken by CSF to fill needle hub (P > 0.05). 


\section{Discussion}

In the present study we observed that Valsalva maneuver significantly reduced the incidence and severity of skin puncture pain associated with lumbar puncture $(\mathrm{P}<0.05)$.

Skin puncture is a painful and distressing factor for any patient undergoing spinal anesthesia. Skin puncture gives a patient both physical as well as psychological trauma. Physiological and psychological factors, both determine the perception of skin puncture pain during any invasive procedure. Psychological factor of fear towards needle poke, unknown unanticipated events and anticipation of pain may affect the pain perception of patient. Pain felt during skin puncture can exaggerate anxiety and physiological reactions to further procedure [8]. Valsalva maneuver has been reported to be effective in attenuating venipuncture pain [9]. Pain attenuation observed during skin puncture in the Valsalva group could have been multifactorial. The observed reduction in venipuncture pain in Valsalva group could possibly because of both distraction component and the sinoaortic baroreceptor reflex arc mediated antinociceptive mechanism [9-11]. Therefore, we planned to evaluate efficacy of Valsalva maneuver to address both physiological and psychological aspect of skin puncture pain felt during spinal anesthesia.

The Valsalva maneuver is performed by forced expiration against the closed glottis and holding this for at least $16 \mathrm{sec}-$ onds [10]. We therefore in the present study performed skin puncture for spinal anesthesia 20 seconds following Valsalva maneuver. Valsalva increases intrathoracic pressure leading to great vessels compression which in turn activates baroreceptor as well as distracts the subject from the immediate environment [8-10]. Valsalva attenuates pain perception through a complex mechanism as proven by pharmacological, neuro-anatomical, electrophysiological and behavioral data suggesting that systems regulating haemodynamics are closely linked to modulators of pain perception [9]. This has been supported by previous studies showing that activation of baroreceptor reflex arc triggers antinociception [9]. The observed reduction in skin puncture pain in Valsalva group could possibly because of both distraction component and the sinoaortic baroreceptor reflex arc mediated antinociceptive mechanism [9-11]. Sinoaortic baroreceptor reflex arc maintains circulatory homeostasis utilizing peripheral receptors and central nervous system through reflex adjustment of parasympathetic and sympathetic efferents to the cardiovascular system. Peripheral activation of sinoaortic baroreceptor reflex arc by cardiovascular stimuli simultaneously involves central nervous system which inhibits pain. Antinociceptive activity of sinoaortic baroreceptor reflex is mediated by three mechanisms like releasing opioid like substance-P into circulation, by secreting vasoconstrictor like noradrenaline increasing blood pressure and by distributing blood towards the heart leading to increased central blood volume [10,11].

Psychological component of pain can be dealt by distracting the patient from the surrounding. Distraction is a congnitive behavioral preparation which acts by manipulating attention framework and by diverting away from pain producing stimulus in the immediate environment $[12,13]$. Distraction not only reduces magnitude of fear, anxiety and pain but also maximizes coping capability of patient to procedural pain [13-15]. Different methods of distraction like cough trick, virtual reality, movies, and balloon inflation have been studied [6-8,13]. Ballon inflation technique in pediatric venipuncture utilizes advantage of both psychological and physiological methods of pain attenuation through performing valsalva manuever [8]. Distraction reduces pain by activating the segmental pain inhibitory pathways [16]. Distraction group utilizes direction of attention method but Valsalva mauever incorporates both attention diversion and physiological methods of pain reduction [8].

In our study, Valsalva group has shown significant reduction in incidence and severity of spinal puncture pain as compared to Distraction and Control group.

Limitations of the present study are lack of double blinding; Cerebrospinal fluid pressure wasn't measured during the study; local anesthetic infiltration of the skin and subcutaneous tissue was not done prior to skin puncture. We did not assess pain due to penetration of subcutaneous tissue, ligaments and ligamentum flavum during spinal puncture. We did not monitor duration of analgesic effect of Valsalva maneuver. Further studies are required in this direction.

In conclusion, we report that Valsalva maneuver performed before spinal anesthesia reduced both incidence and severity of spinal puncture pain as compared to Distraction and control group. This magnitude of reduction could be secondary to reduction in both psychological and physiological component of pain by performing Valsalva maneuver. Distraction group was found to be inferior to Valsalva group in reducing pain as it utilizes only attention diversion to reduce pain. Distraction group had shown reduction in severity of pain as compared to control group. We further suggest that Valsalva should be performed routinely in all ASA I and II patients undergoing spinal anesthesia as it is an inexpensive, easy to perform, painless and non-pharmacological method of pain attenuation during spinal puncture. 


\section{References}

1. Gajraj NM, Sharma S, Souter AJ, Pole Y, Sidawi E. A survey of patients who refuse regional anesthesia. Anesth Analg 1994; 78 : S126.

2. Morris RW, Whish DK. A controlled trial of pain on skin infiltration with local anesthetics. Anaesth Intensive Care 1984; 12: 113-4.

3. Morris R, McKay W, Mushlin P. Comparison of pain associated with intradermal and subcutaneous infiltration with various local anesthetic solutions. Anesth Analg 1987; 66: 1180-2.

4. Koscielniak-Nielsen Z, Hesselbjerg L, Brushøj J, Jensen MB, Pedersen HS. EMLA patch for spinal puncture. A comparison of EMLA patch with lignocaine infiltration and placebo patch. Anesthesia 1998; 53: 1218-22.

5. Patterson P, Hussa AA, Fedele KA, Vegh GL, Hackman CM. Comparison of 4 analgesic agents for venipuncture. AANA J 2000 ; 68 : 43-51.

6. Usichenko TI, Pavlovic D, Foellner S, Wendt M. Reducing venipuncture pain by a cough trick: a randomized crossover volunteer study. Anesth Analg 2004; 98: 343-5.

7. Wismeijer AA, Vingerhoets AJ. The use of virtual reality and audiovisual eyeglass systems as adjunct analgesic techniques: a review of the literature. Ann Behav Med 2005; 30: 268-78.

8. Gupta D, Agrawal A, Dhiraaj S, Tandon M, Kumar M, Singh RS, et al. An evaluation of balloon inflation on venous cannulation pain in children: a prospective, randomized, controlled study. Anesth Analg 2006; 102: 1372-5.

9. Agarwal A, Sinha PK, Tandon M, Dhiraaj S, Singh U. Evaluating the efficacy of the valsalva maneuver on venous cannulation pain: a prospective, randomized study. Anesth Analg 2005; 101: 1230-2.

10. Englstrom JW, Martin JB. Disorders of the autonomic nervous system. In: Principals of internal medicine. Edited by Braunwald E, Fauci AS: New York, McGraw-Hill. 2001, pp 2416-21.

11. Randich A, Maixner W. Interaction between cardiovascular and pain regulatory systems. Neurosci Biobehav Rev 1984; 8: 343-67.

12. McCaul KD, Malott JM. Distraction and coping with pain. Psychol Bull 1984; 95: 516-33.

13. Cohen LL. Reducing infant immunization distress through distraction. Health Psychol 2002; 21: 207-11.

14. Cohen LL. Behavioral approaches to anxiety and pain management for pediatric venous access. Pediatrics 2008; 122 Suppl 3: S134-9.

15. Cohen LL, Bernard RS, Greco LR, McClellan CB. A child-focused intervention for coping with procedural pain: are parent and nurse coaches necessary? J Pediatr Psychol 2002; 27: 749-57.

16. Ong EL, Lim NL, Koay CK. Towards a pain-free venipuncture. Anaesthesia 2000; 55: 260-2. 